\title{
Tocilizumab is efficacious in active RA as little as 1 week after treatment
}

The anti-IL-6 receptor antibody tocilizumab could provide clinical benefit as soon as 1 week after treatment initiation in patients with RA, according to new data from a 24-week phase IIIb trial.

This randomized, placebo-controlled, multicenter study, led by Yusuf Yazici of the New York University Hospital for Joint Diseases, included 619 patients with moderate-to-severe active RA and a previous inadequate response to DMARDs. Tocilizumab was considerably more efficacious than placebo as early as 4 weeks after treatment and up to week 24 , as revealed by multiple outcome measures. $30.1 \%$ of the tocilizumab group $(n=412)$ attained the primary endpoint of an ACR50 response, compared with $11.2 \%$ of the placebo group $(n=207)$. These data add to previous evidence of the efficacy of tocilizumab in the treatment of RA.

What made this trial unique was that a subset of 62 patients were assessed at 3 and 7 days after commencement of treatment, in addition to the regular assessment time points, "specifically to measure how early the response can be seen," states Yazici. Laboratory markers, DAS28 and patient-reported assessments had all improved by day 7 to a greater degree in the tocilizumab group $(n=40)$ than the placebo group $(n=22)$, whereas other physician-reported assessments had not. These findings suggest "that there is good early response and patientreported outcomes seem to be better at this early time point in differentiating drug response from placebo response," concludes Yazici. It remains to be seen whether this early response is predictive of long-term clinical benefit.

\section{David Killock}

Original article Yazici, Y. et al. Efficacy of tocilizumab in patients with moderate to severe active rheumatoid arthritis and a previous inadequate response to disease-modifying antirheumatic drugs: the ROSE study. Ann. Rheum. Dis. doi:10.1136/ard.2010.148700 